\title{
An Ethical Framework for Automated, Wearable Cameras in Health Behavior Research
}

\author{
Paul Kelly, MSc, Simon J. Marshall, PhD, Hannah Badland, PhD, Jacqueline Kerr, PhD, \\ Melody Oliver, PhD, Aiden R. Doherty, PhD, Charlie Foster, PhD
}

\begin{abstract}
Technologic advances mean automated, wearable cameras are now feasible for investigating health behaviors in a public health context. This paper attempts to identify and discuss the ethical implications of such research, in relation to existing guidelines for ethical research in traditional visual methodologies. Research using automated, wearable cameras can be very intrusive, generating unprecedented levels of image data, some of it potentially unflattering or unwanted. Participants and third parties they encounter may feel uncomfortable or that their privacy has been affected negatively. This paper attempts to formalize the protection of all according to best ethical principles through the development of an ethical framework. Respect for autonomy, through appropriate approaches to informed consent and adequate privacy and confidentiality controls, allows for ethical research, which has the potential to confer substantial benefits on the field of health behavior research.
\end{abstract}

(Am J Prev Med 2013;44(3):314-319) @ 2013 American Journal of Preventive Medicine

\section{Introduction}

W ith improvements in device technology and data storage, research that employs visual data capture increasingly is being employed to investigate health behaviors such as exercise and diet. ${ }^{1-4}$ Although image-based research (e.g., using photography) has existed for more than 100 years, wearable devices that capture images and allow observation and recording of an individual's health behaviors only have been practical and feasible in the past decade. ${ }^{5,6}$ The current paper discusses the ethical issues of research using an automated, wearable camera, with a focus on the Microsoft SenseCam.

When SenseCam research has been presented at scientific meetings, the issue of ethics has always been raised. Audiences generally are skeptical that such image data can be collected within accepted ethical frameworks. Some perceive it as highly intrusive; others cannot envi-

From the British Heart Foundation Health Promotion Research Group (Kelly, Doherty, Foster), Department of Public Health, University of Oxford, United Kingdom; the Department of Family and Preventive Medicine (Marshall, Kerr), University of California San Diego, La Jolla, California; Centre for Physical Activity and Nutrition (Oliver), Auckland University of Technology, New Zealand; and McCaughey VicHealth Centre for the Promotion of Mental Health and Community Wellbeing (Badland), University of Melbourne, Victoria, Australia

Address correspondence to: Paul Kelly, MSc, Department of Public Health, Old Road Campus, Headington, Oxford, OX3 7LF, United Kingdom.E-mail: paul.kelly@dph.ox.ac.uk.

$0749-3797 / \$ 36.00$

http://dx.doi.org/10.1016/j.amepre.2012.11.006 sion the data being collected without breaching accepted ethical practice and codes of conduct.

To date, the current authors have been involved in six successful IRB applications for health-related wearable-camera research and are often contacted for ethical guidance by researchers wishing to use such techniques at their own institutions. This paper discusses how issues involving automated, wearable cameras relate to existing ethical frameworks for visual research. A framework for future ethical practice using such cameras is proposed.

\section{Image-Based Research}

Guidelines have been developed for ethical research practice using images of participants or people they encounter, notably by the British Sociological Association (BSA) in 2006 and the Economic and Social Research Council (ESRC) in 2008. ${ }^{7}$ The central theme of both sets of guidelines is the importance of consent (preferably written) both to participation and the ways the image data will be used. ${ }^{6,8}$

These guidelines were developed from the philosophy of the non-consequentionalist approach, which is related to principalist approaches, commonly used in public health research. ${ }^{8,9}$ They are based on the following principles: respect for autonomy, which relates to issues of voluntariness, informed consent, confidentiality, and anonymity; beneficence, which concerns the responsibility to do good; non-maleficence, which involves the re- 
sponsibility to avoid harm; and justice, which encompasses the importance of the benefits and burdens of research being distributed equally. ${ }^{8,9}$ These are the four main principles of ethical research as considered by IRBs.

Prosser et al. ${ }^{7,8}$ subsequently codified these guidelines for visual research as follows:

- researchers should strive to protect the rights, privacy, dignity, and well-being of those that they study;

- research should be (as far as possible) based on voluntary informed consent;

- personal information should be treated confidentially and participants anonymized unless they choose to be identified;

- research participants should be informed of the extent to which anonymity and confidentiality can be ensured in publication and dissemination and of the potential reuse of data.

\section{Automated, Wearable Cameras As a New Technique in Visual Research}

Four common types of visual research data exist: (1) found or existing data (e.g., photo albums not originally created for research purposes); (2) researchercreated research data; (3) respondent-created research data; and (4) representations; these can take forms such as photos, videos, films, drawings, and graphical representations. ${ }^{7,8}$ The daily capture of thousands of first-person point-of-view images from an automated, wearable camera is a new technique that has emerged from the field of digital life-logging. ${ }^{10}$ SenseCam, worn on a lanyard around the neck, is an example of such a device. ${ }^{5}$ In public health, it currently is being utilized to study active travel, physical activity, sedentary behavior, and diet.

In traditional participant-created data techniques, researchers gave cameras to participants and asked them to take photographs at certain times of certain things. Techniques with cameras such as SenseCam elicit a shift from this researcher-centric construction of the social world to that of the participants. ${ }^{7}$ Image collection is passive rather than purposive, allowing researchers to see aspects of participants' lives they might otherwise not gain insight or access to.

The nature of this type of research raises a number of novel issues with ethical implications. These issues have been identified in two ways: (1) from experience of extensive field testing of SenseCam and six pilot studies, utilizing participant-satisfaction interviews and IRB application feedback, and (2) from a theoretic examination of the existing public health and image research literature, considering the new and specific characteristics of SenseCam.

\section{Image Volume Compared to Traditional Photography}

Automated, wearable cameras collect many more images than traditional photography (2000-3000 per 12 hours of wear). Compared with traditional photography, automated, wearable cameras collect considerably more information and are therefore potentially more intrusive than 30 self-selected participant-captured images taken with a camera. In turn, the privacy of the participant, a key component of autonomy, is threatened. In order to maintain respect for autonomy, this fact must be explained clearly to participants before the collection of informed consent.

With far more image information, confidentiality and security of collected data take on greater importance because the potential harm from data becoming public is greater. Confidentiality can be protected by configuring devices and using specialist viewing software to make the images accessible only to the research team. In this way, lost devices do not pose such a risk to privacy. As with any visual research, collected images should be stored securely and password-protected, according to national regulations (e.g., the UK Data Protection Act). ${ }^{6}$

The use of the collected images must be in accordance with protection of privacy, confidentiality, and anonymity. No image that identifies a participant by place, context, or other factor should be disseminated or shared without participants' express consent. In health behavior research, images are aggregated to investigate behavior and do not have to be published to support the data. By contrast, traditional visual research methodologies sometimes use photos for illustration of social norms or events. If the images taken for such research remain within the private domain of the researcher, privacy is preserved more easily than if the images are used publically.

In the event that images are used in any paper, publication, or public setting, faces and identifying features should be obscured. This is especially important in light of recent technologies such as Facebook's facial recognition software. Participant information should be explicit about data retention, and the possible secondary analyses that may take place.

\section{Passive Image Capture}

Image capture with an automated, wearable camera is passive, not purposive; the camera records images independent of any action by the wearer. In traditional visual research methods, the image is posed or chosen by the participant. In research with automated, wearable cameras, the image is taken automatically, meaning the participant does not have as much control over its timing and content. The images captured may be unwanted or unflattering; for example, adjusting clothing in a mirror, 
grabbing a handful of food from the fridge, or browsing social networking sites while at work. This loss of control can be interpreted as a loss of autonomy, and therefore this must be made explicit at the informed consent stage.

The participant can regain some autonomy in three ways. First, SenseCam is fitted with a privacy button that prevents image capture for a 7 -minute period and is designed for activities such as visiting the bathroom. Second, participants should be expressly advised that they may remove the camera (or turn it around so the lens faces inward), whenever they wish, for any reason, without having to provide an explanation to the research team. For example, they may wish to remove it when trying on clothes, doing online banking, or in social situations such as waiting outside school for children.

Although this practice reduces the amount of data collected and may bias the context of these behaviors, it is vital for participant autonomy. Moreover, in the context of 3 days of behavioral research, losing 10 minutes of data from time to time will not devalue the research substantially. Finally, participants should be given the option to review and delete images at the end of data collection.

There also may be settings where photography is prohibited, such as some workplaces, banks, swimming pools, and airport security. Participants should be advised to remove the device before entering these settings to avoid challenge or incident. Pocket-sized information cards can be provided to help participants explain the device if needed. As a result of these and other settings, there may be some populations that are not suitable to be included in this type of research. Specific examples include medical or care providers interacting with patients, workers at military institutions, or those at workplaces with high levels of commercial secrecy.

\section{Inappropriate or Unwanted Images}

The participant may forget they are wearing the camera and capture images and information they would not wish or choose to have on record. While privacy buttons exist and wearable cameras can be removed, it is not uncommon for participants to report that they forgot they were wearing the unit. Therefore, the participant inadvertently might collect inappropriate images, such as going to the bathroom, Internet habits, reading confidential documents, getting undressed, or being in the presence of family members who are dressing. As part of the informed consent process, and to reduce any anxiety related to unintended image capture, the participant should be made aware that unwanted images might be captured and that they will be given the opportunity to review and delete them (in private if requested) before the researcher views them.
Participants often enjoyed reviewing their own images and requested copies of the images. Images should not be given to participants, as once the image has been "given away" by the researcher they cannot control its use (e.g., on social networking sites), or the context in which it is displayed. There is consensus that when visual data are being used solely for observation or elicitation purposes then issues of consent are relatively unproblematic; it is the dissemination and publication of images that create the most complex issues. ${ }^{8}$

\section{Confidentiality}

Confidentiality may not always be possible. Once the participant has reviewed and deleted any images, those remaining will be retained as data. At this point, a legal issue arises. Allen et al. ${ }^{11}$ recently stated that "the law may not permit privacy." If the images depict any illegal activities, according to national regulation, the researcher may be under legal and professional obligation to breach confidentiality and pass on image data to appropriate authorities. Examples of illegal activities that plausibly could be captured are texting while driving, drinking in a bar prior to driving home, or taking illicit substances. Images of criminal damage, sexual violence, and hate crime are not protected by confidentiality and may be liable to subpoena by a court. ${ }^{6}$

There is a greater risk of participants incriminating themselves by wearing an automated camera device than through traditional methods because of the issues described above. Research participants must be informed of this. In the U.S., the NIH can provide a Certificate of Confidentiality that prevents research data from being subpoenaed, and the obligation to report events such as abuse is often limited to certain groups such as medical employees or clinical psychologists. Despite this, the authors believe that researchers are obliged to report abuse.

There is also the possibility that the participant might be involved in an incident during data collection requiring legal intervention (e.g., violence or a road traffic accident). Digital records could be seized and used by governments and law enforcement agencies, for prosecutions to add context and remove ambiguity in these events. ${ }^{12}$ In these instances, privacy of research participants cannot be guaranteed. As a result, researchers must make sure participants are fully informed from the outset that certain images would be subject to these laws to preserve autonomy.

\section{Third Parties}

Third parties who encounter the wearer have their image captured without the opportunity to provide informed consent. Over the course of a typical day, a participant may encounter many people. These people may be pho- 
tographed, knowingly or unknowingly, and will not have the opportunity to provide informed consent. In traditional methods, there would likely be a single image and those nearest the photographer (so most prominent in the image) would have an understanding of being "photographed"; this is not the case for automated, wearable cameras.

These third parties can include family members, cohabitants, friends, colleagues, coworkers, acquaintances, and individuals unknown to the participant. Each is discussed below according to the specific issues surrounding privacy and autonomy. For all cases, issues of confidentiality, data security, and image use should be treated as discussed in the previous sections.

Family members, cohabitants, and friends. Existing guidelines for visual research recognize that privacy includes reasonable expectations of where you will not be photographed unknowingly and this should be considered the case for third parties in their own home. ${ }^{6,8} \mathrm{Al}-$ though it is not necessary to obtain written informed consent from all family members and cohabitants, the participant should seek their verbal permission prior to wearing an automated camera in the home. Researchers should provide the necessary information to help participants explain that images will be recorded, but securely stored and not disseminated. The privacy of friends and acquaintances also should be respected. When prior verbal permission is not practical, verbal permission and explanations on first contact will respect their autonomy.

Colleagues and coworkers. Workplaces vary considerably in terms of number of employees, context, relationships with coworkers, and interaction with the public. Consequently, the appropriateness of an automated, wearable camera likely would differ among workplaces (e.g., an office, shop, and hospital). The key issue is about respecting privacy, and each setting should be assessed by the researcher before issuing devices. When the workplace involves interaction with children (e.g., teachers, other school workers, or daycare workers), parents should be informed of the research.

Studies in leisure-time physical activity or active travel may not necessitate that participants wear the device at work. However, research focusing on sedentary behavior or diet may require devices to be worn during working hours. In such cases, participants should seek verbal permission from managers or supervisors, and should inform direct coworkers about the device. If requested by these individuals, the device should be removed. Individuals working with clients should be advised that the study is not appropriate for them.

Strangers and the general public. Existing guidelines state that when taking images of individuals and groups in public spaces, it is not practical or necessary to obtain informed consent unless the images are published or disseminated in such a way that they can be recognized. ${ }^{6,8}$ However, participants should remove devices if they find themselves recording strangers where privacy reasonably might be expected, such as gym changing rooms. Consideration also should be given to research in certain cultural settings in which photography is disapproved of or considered inappropriate (e.g., aboriginal communities). ${ }^{6}$ Clear, written guidance and examples of these potential situations should be given by researchers to the participants when the devices are distributed.

There is risk of burden or harm to the participant (from the principle of non-maleficence) when wearing devices in free-living situations. Possible scenarios include the following: the participant is questioned by (potentially hostile or suspicious) third parties who object to unsolicited image recording, and the participant attracts threat to his or her safety (e.g., mugging). In the first case, it was found that preparing the participant with a simple statement explaining the study purpose and device and concluding with the offer to remove it reduces participant and third party burden. In the second case, instructing the participants to remove the device if they ever feel uncomfortable or threatened (e.g., walking home late at night) is recommended to reduce the risk of related harm. Participants also should be told to inform third parties that they can request deletion of images they are in by asking the participant to inform the research team or contacting them directly.

Some third parties will not know they have been photographed. This means that such research has parallels to covert research, which is subject to serious ethical and legal issues. ${ }^{6}$ However, the distinction is that research with automated, wearable cameras is not directly about the third parties. Nonetheless, whether they know they have been photographed or not, respect for autonomy directs that the privacy of third parties must be protected. If their behavior is investigated as influencing that of the participant (e.g., eating or drinking in a group setting), their anonymity should be preserved, and no image that identifies them should be published without consent.

\section{Image Viewing by a Large Team of Researchers}

A participant may prefer that only the researcher(s) they have met and spoken to see their images. However, a typical study of this type (usually around 50 participants) with data collected for 3 days will generate nearly 500,000 images. This means a team of researchers is needed to code and handle the data. At informed consent, participants should be made aware that a team of trained researchers will be viewing their images. 
Further, all researchers who come into contact with the images should be trained and instructed appropriately in correct, ethical use of the data. General ethical training required by most research institutes does not go into detail about the above risks mentioned and the seriousness of breaches of confidentiality. Researchers coding image data should be reminded to not discuss the content with anyone outside of the team, to not identify anyone they recognize in the images, to store the images safely, and to be aware of how sensitive the data are. A training manual for researchers who will be in contact with image data has been developed at the
University of California San Diego (available from authors on request).

\section{Other Issues}

This paper focuses on observational studies with volunteer participants. Studies that utilize automated, wearable cameras and their images to elicit behavior change will have slightly different issues (e.g., guided reflection or discussion in focus group settings). Studies in vulnerable populations question the extent to which participants can give informed consent, and consent of carers or significant others may be required. ${ }^{13}$

Table 1. Ethical guidelines for the use of automated, wearable cameras in observational health behavior research

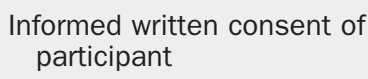

Privacy and confidentiality

Non-maleficence

Autonomy of third parties
Participant information should explicitly detail the following:

how many images and how much information will be collected

the nature and type of data that can be collected by wearing an automated, wearable camera (images will depict where you go, what you do, and for how long) with examples participants can forget they are wearing the device and record unwanted and unflattering images with examples provided (e.g., bathroom visits, online banking)

data of illegal activities may not be protected by confidentiality and may be passed to law enforcement depending on the national law and nature of the activity

no individual will be identifiable in any research dissemination without their consent participants will have the opportunity to view (and delete if necessary) their images in privacy participants are able to remove the device or temporarily pause image capture whenever they wish

participants will not get copies of their images

a team of specifically trained researchers will have access to the image data

Devices should be configured so that data can only be retrieved by the research team. It should be impossible for participants or third parties who find devices to access images

Data should be stored according to national data protection regulations

Identifying images should not be used without express consent of those individuals who are depicted

Devices should be configured to allow participants to cease recording for short periods. Participants should be allowed to remove the device at any time, with examples of where this might be appropriate (e.g., airport security)

Appropriate training should be provided for all those in the research team who have contact with the image data

Participants should be prepared for questions by the public with a short sentence that explains the device and concludes with an offer to remove if they are feeling uncomfortable

Participants should be instructed to remove device in any situation where it is attracting unwanted attention, or they feel threatened or uneasy wearing the device

Participants should seek verbal permission from family members and cohabitants before study commencement

Participants should seek verbal permission of workplace managers or supervisors. If possible, this should be prior to study commencement, but in reality may be a rolling process. Appropriateness of device to work setting should be assessed by researcher

Participants should inform friends and acquaintances of device when encountered and offer to remove device if they are uncomfortable

Participants should be told to inform third parties that they also can request image deletion by asking the participant to inform the research team, or contacting them directly

The privacy and anonymity of third parties must be protected; no image that identifies them should be published without their consent

Photography is inappropriate in some cultural settings and automated, wearable cameras should not be used in these instances 


\section{Operating Ethical Framework}

Based on the ethical issues discussed above, a checklist has been produced for research using automated, wearable cameras in free-living observational studies (Table 1). The authors see this as an initial attempt to provide a framework for such research and acknowledge that there may be other important issues that are not covered or as yet known about; as new challenges and issues emerge, frameworks and ways of working should be revised and adapted to ensure ethical research and best practice.

\section{Conclusion}

Technologies often evolve faster than legal and ethical systems can respond and unforeseen ethical issues emerge; healthcare technologies are a heterogeneous group, and ethical issues should be assessed separately and thoroughly. ${ }^{12,14}$ Research using automated, wearable cameras by its nature can be very intrusive, and participants and third parties may still feel uncomfortable. This paper attempts to formalize the protection of all according to best ethical principles through the development of an ethical framework.

Automated, wearable cameras passively generate more image data than ever before, some potentially unflattering or unwanted. Respect for autonomy, through appropriate approaches to informed consent and adequate privacy and confidentiality controls, allows for ethical research that has the potential to confer substantial benefits to the field of health behavior research. ${ }^{15}$

This work was supported by Microsoft Research through its $\mathrm{PhD}$ Scholarship Programme; the British Heart Foundation under grant number 021/P\&C/core/2010/HPRG; and the Irish Health Research Board under grant number MCPD/2010/12.

No financial disclosures were reported by the authors of this paper.

\section{References}

1. Sun M, Fernstrom JD, Jia W, et al. A wearable electronic system for objective dietary assessment. J Am Diet Assoc 2010;110(1):45-7.

2. Arab L, Estrin D, Kim DH, Burke J, Goldman J. Feasibility testing of an automated image-capture method to aid dietary recall. Eur J Clin Nutr 2011;65(10):1156-62.

3. Kelly P, Doherty A, Berry E, Hodges S, Batterham AM, Foster C. Can we use digital life-log images to investigate active and sedentary travel behaviour? Results from a pilot study. Int J Behav Nutr Phys Act 2011;8:44.

4. Zhang H, Li L, Jia WY, Fernstrom JD, Sclabassi RJ, Sun MG. Recognizing physical activity from ego-motion of a camera. 2010 Annual International Conference of the Engineering in Medicine and Biology Society (EMBC). Conf Proc IEEE Eng Med Biol Soc 2010;2010:5569-72.

5. Hodges S, Williams L, Berry E, Izadi S, Srinivasan J, Butler A, et al. SenseCam: A retrospective memory aid. Ubicomp 2006: Ubiquitous Computing, Proceedings 2006;4206:177-93.

6. British Sociological Association. Statement of ethical practice for the British Sociological Association-Visual Sociology Group. Durham: BSA, 2006.

7. J Prosser, A Loxley. ESRC National Centre for Research Methods review paper: introducing visual methods. Southampton: National Centre for Research Methods, 2008.

8. Wiles R, Prosser J, Bagnoli A, Clark A, Davies K, Holland S, et al. Visual ethics: ethical issues in visual research. Southampton: National Centre for Research Methods, 2008.

9. Papademas D. Editor's introduction: ethics in visual research. Visual Studies 2004;19(2):122-6.

10. Mann S. Wearable computing: a first step toward personal imaging. Computer 1997;30(2):25-31.

11. Allen AL. Unpopular privacy: what must we hide? New York: Oxford University Press, 2011.

12. O'Hara K, Tuffield M, Shadbolt N. Lifelogging: privacy and empowerment with memories for life. Identity Informa Soc 2008; 1(1):155-72.

13. Novitzky P, Gordijn B, Jacquemard T, O’Brolcháin F. Dementia ambient care: multi-sensing monitoring for intelligent remote management and decision support: ethical literature review. Dublin, Ireland, 2012.

14. Hofmann B. Ethical challenges with welfare technology: a review of the literature. Sci Eng Ethics 2012;5:5.

15. European Network and Information Security Agency. Life logging risk assessment; Final report. Heraklion, Greece: ENISA, 2011. 